\title{
Analysis of circulating tumor DNA: The next paradigm shift in detection and treatment of lung cancer
}

\author{
David S. Schrump, MD, MBA, and Julie A. Hong, MS
}

\footnotetext{
From the Thoracic Epigenetics Section, Thoracic and Oncologic Surgery Branch, Center for Cancer Research, National Cancer Institute, Bethesda, Md.

Disclosures: Authors have nothing to disclose with regard to commercial support.

Received for publication Jan 31, 2018; accepted for publication Jan 31, 2018.

Address for reprints: David S. Schrump, MD, MBA, Thoracic Epigenetics Section, Thoracic and Oncologic Surgery Branch, Center for Cancer Research, National Cancer Institute, Building 10, 4-3942, 10 Center Dr, Bethesda, MD 20892 (E-mail: David_Schrump@nih.gov).

J Thorac Cardiovasc Surg 2018;155:2632-3

0022-5223/\$0.00

Published by Elsevier Inc. on behalf of The American Association for Thoracic Surgery https://doi.org/10.1016/j.jtcvs.2018.01.060
}

The discovery that specific activating mutations confer sensitivity of lung cancer cells to tyrosine kinase inhibitors ushered in a new era of precision therapy and constituted the first major paradigm shift in the diagnosis and treatment of lung cancer in several decades. ${ }^{1,2}$ The National Cancer Institute-sponsored randomized trial demonstrating that computed tomographic screening reduces lung cancerspecific mortality prompted the second paradigm shift in lung cancer care. $^{3}$ US Food and Drug Administration approval of immune checkpoint inhibitors as first- or second-line therapy for non-small cell lung cancer was the third major paradigm shift in lung cancer treatment. ${ }^{4}$ More recently, the rapid development of next-generation sequencing (NGS) of circulating tumor DNA (ctDNA) is promoting the fourth paradigm shift in lung cancer care in less than 15 years..$^{5-7}$

In this issue of the Journal, Isbell and colleagues ${ }^{8}$ review the current status of ctDNA analysis in patients with lung cancer. The review is well balanced and highlights results of several leading publications in this rapidly evolving field. The central themes emerging from these studies are that levels of ctDNA vary among patients with cancer of similar tissue histologic type and stage; however, in any given patient with detectable ctDNA, there appear to be good correlations between ctDNA levels and tumor burden, prognosis, and survival. Current data suggest that patients with detectable ctDNA at diagnosis have worse outcomes than those with no detectable ctDNA. Furthermore, patients whose ctDNA levels persist after definitive treatment of their neoplasms are significantly more likely to have early disease recurrence and death than are patients whose ctDNA levels are undetectable after treatment (Figure 1). Molecular progression appears to precede clinical recurrence and progression.

NGS of ctDNA allows the detection of cancer-associated driver mutations, such as EGFR or RAS, as well as "passenger" mutations that do not impact growth or metastatic cation errors.

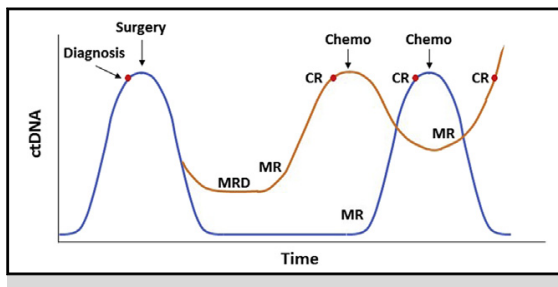

Circulating tumor DNA and recurrence.

Central Message

Circulating tumor DNA provides a noninvasive means of diagnosis and surveillance of lung cancer. This evolving technology represents the next paradigm shift in lung cancer care.

See Article page 2628.

potential of the cancers but are nonetheless useful for disease surveillance. For example, by using cancerpersonalized profiling by deep sequencing techniques to monitor disease status in patients with lung cancer after definitive standard-of-care treatment, Chaudhuri and associates $^{6}$ identified driver mutations in $35 \%$, passenger-only mutations in $35 \%$, and both types of mutations in $30 \%$ of patients with detectable ctDNA. The presence of detectable mutations during surveillance preceded clinical recurrence or progression by approximately 5 months.

NGS of ctDNA may also reveal actionable mutations not detected by fine-needle aspiration of heterogenous solid tumors, as well as clonal evolution in response to therapy. Furthermore, NGS of ctDNA may identify cancers with high mutational burdens with the potential to respond to immune checkpoint inhibitors, ${ }^{6}$ and decreases in ctDNA correlate with and precede clinical regressions in lung cancer patients who receive these agents.

One limitation of the existing technology is that ctDNA is not detected in all patients with lung cancer; another limitation is that the amount of tumor required for reliable detection of ctDNA is approximately $10 \mathrm{~cm}^{3}$ at a threshold of $0.1 \%$ variable allele frequency. ${ }^{5}$ As such, ctDNA analysis currently is not applicable for use in lung cancer screening, nor can ctDNA analysis reliably establish or exclude the presence of early invasive cancers in ground glass opacities. Theoretically, whole-genome amplification could increase input DNA levels for NGS, but it could also enhance the potential for false-positive sequencing results due to amplifi- 


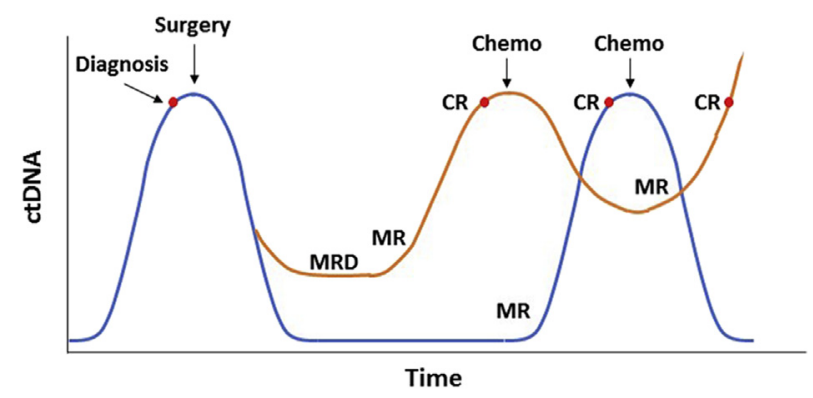

FIGURE 1. Circulating tumor ( $c t D N A$ ) levels reflect disease burden and prognosis in patients with lung cancer. The ctDNA levels increase as occult lung cancer progresses, diagnosis is established, and treatment is initiated. In patient 1 (blue line), circulating tumor DNA levels fall below the detection threshold and remain undetectable until tumor recurs. Molecular recurrence $(M R)$ precedes clinical recurrence $(C R)$. Chemotherapy is initiated, at which point circulating tumor DNA levels fall again, indicating a chemosensitive tumor. In contrast circulating tumor DNA levels fall but remain detectable in patient 2 (yellow line), who has more rapid molecular as well as clinical recurrence of a treatment-resistant cancer in the setting of molecular residual disease (MRD).

Although recent studies in high-tier journals are encouraging, ${ }^{5-7}$ additional, larger prospective trials are necessary to establish the reliability, reproducibility, and accuracy of ctDNA analysis in lung cancer patients. The American Association for Thoracic Surgery and Thoracic Surgery
Oncology Group-sponsored multicenter trial examining the utility of ctDNA as prognostic and predictive biomarkers in patients with lung cancer undergoing induction therapy before surgery is a big step in the right direction.

\section{References}

1. Lynch TJ, Bell DW, Sordella R, Gurubhagavatula S, Okimoto RA, Brannigan BW, et al. Activating mutations in the epidermal growth factor receptor underlying responsiveness of non-small-cell lung cancer to gefitinib. N Engl J Med. 2004; 350:2129-39.

2. Rajan A, Schrump DS. Precision therapy for lung cancer: tyrosine kinase inhibitors and beyond. Semin Thorac Cardiovasc Surg. 2015;27:36-48.

3. National Lung Screening Trial Research Team; Aberle DR, Adams AM, Berg CD, Black WC, Clapp JD, Fagerstrom RM, et al. Reduced lung-cancer mortality with low-dose computed tomographic screening. $N$ Engl J Med. 2011;365:395-409.

4. Assi HI, Kamphorst AO, Moukalled NM, Ramalingam SS. Immune checkpoint inhibitors in advanced non-small cell lung cancer. Cancer. 2018;124: 248-61.

5. Abbosh C, Birkbak NJ, Wilson GA, Jamal-Hanjani M, Constantin T, Salari R, et al. Phylogenetic ctDNA analysis depicts early-stage lung cancer evolution. $\mathrm{Na}$ ture. 2017;545:446-51. Erratum in: Nature. 2018;554:264.

6. Chaudhuri AA, Chabon JJ, Lovejoy AF, Newman AM, Stehr H, Azad TD, et al Early detection of molecular residual disease in localized lung cancer by circulating tumor DNA profiling. Cancer Discov. 2017;7:1394-403.

7. Goldberg SB, Narayan A, Kole AJ, Decker RH, Teysir J, Carriero NJ, et al. Early assessment of lung cancer immunotherapy response via circulating tumor DNA. Clin Cancer Res. January 12, 2018 [Epub ahead of print].

8. Isbell JM, Jones DR, Li BT. Circulating tumor DNA: a promising biomarker to guide post-operative treatment and surveillance of non-small cell lung cancer. $J$ Thorac Cardiovasc Surg. 2018;155:2628-31. 